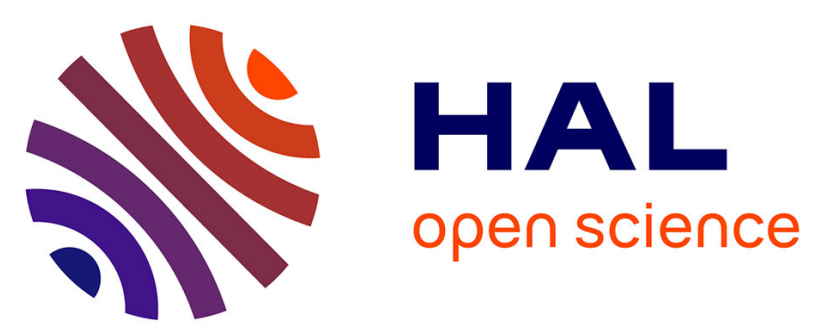

\title{
Design of a robust polytopic unknown input observer for FDI: Application for systems described by a multi-model representation
}

Mickael Rodrigues, Didier Theilliol, Dominique Sauter

\section{- To cite this version:}

Mickael Rodrigues, Didier Theilliol, Dominique Sauter. Design of a robust polytopic unknown input observer for FDI: Application for systems described by a multi-model representation. 44th IEEE Conference on Decision and Control and European Control Conference, CDC-ECC'05, Dec 2005, Séville, Spain. pp.6268-6273. hal-00364786v2

\section{HAL Id: hal-00364786 \\ https://hal.science/hal-00364786v2}

Submitted on 2 Mar 2009

HAL is a multi-disciplinary open access archive for the deposit and dissemination of scientific research documents, whether they are published or not. The documents may come from teaching and research institutions in France or abroad, or from public or private research centers.
L'archive ouverte pluridisciplinaire $\mathbf{H A L}$, est destinée au dépôt et à la diffusion de documents scientifiques de niveau recherche, publiés ou non, émanant des établissements d'enseignement et de recherche français ou étrangers, des laboratoires publics ou privés. 


\title{
Design of a Robust Polytopic Unknown Input Observer for FDI: Application for Systems described by a Multi-Model Representation
}

\author{
Mickaël Rodrigues, Didier Theilliol and Dominique Sauter
}

\begin{abstract}
The main goal of this paper consists in fault diagnosis of systems represented by a multi-model approach. Partial knowledge of the system representation around operating points must allow to obtain its dynamic behaviour under modeling errors consideration. The goal of this paper is to decouple modeling errors through a wide operating range by a dedicated polytopic Unknown Input Observer $(\mathcal{U I O})$. The robust polytopic observer allows an optimization of modeling errors matrix distribution and permits to develop a fault diagnosis strategy on a wide operating range. Polytopic observer stability is guaranteed by pole assignment established through Linear Matrix Inequality $(\mathcal{L} \mathcal{M} \mathcal{I})$. The effectiveness and performances of the method are illustrated in simulation considering a CSTR.
\end{abstract}

\section{INTRODUCTION}

The increasing demands for good performance and higher standards of safety and reliability result in more emphasis on fault diagnosis. Fault detection and isolation (FDI) refers to the task of inferring the occurrence of faults in a process and finding the root causes of the faults. Fault diagnosis based on analytical models is developed for exact and uncertain linear mathematical description of the system, several books are dedicated to these topics: [1] and [2]. FDI on nonlinear systems remains a challenge due to the problem of discriminating between disturbances and faults through a wide range of operating conditions. Different techniques based on an exact knowledge of the nonlinear system allow to generate residuals insensitive to fault by specific decoupling methods [3], [4] or geometric approach [5], [6].

An attractive alternative to nonlinear modeling problem is to use a multi-linear model approach. This approach is successfully used for some nonlinear systems in control field but rarely in FDI, and consists in partitioning the operating range of a system into separate regions in order to synthesize a global representation. The reader could refer to [7] for a global review on multiple models strategy, and also for well developed identification method and modeling problems. A polytopic representation is sometimes used in multi-model for nonlinear systems modeling as for example in fault-free case in [8], [9], [10] and [11], and in faulty case in [12]. Multiple Model Adaptive Estimation (MMAE) [13] or Interacting Multiple Model (IMM) [14] introduce a multi-model approach for FDI, but these techniques are developed around an unique operating point $(\mathcal{O P})$. Indeed,

This work was not supported by any organization

M. Rodrigues, D. Theilliol and D. Sauter are with Centre de Recherche en Automatique de Nancy - CNRS UMR 7039, Université Henri Poincaré, BP 239 - 54506 Vandoeuvre Cedex France. Phone: +33 383684465 - Fax: +33 383684462 mickael.rodrigues@cran.uhp-nancy. fr these methods consider a particular multi-model approach where each model is dedicated to a specified fault. A fuzzy logic development is also mentioned in [1] for diagnosis but only with Kalman or Luenberger filters which do not permit to deal with modeling errors. Moreover, the activation functions for each model may be not robust to faults.

This paper addresses a different method that could allow to detect both actuator or sensor fault in nonlinear systems which are known and exploited around few local models, i.e. we suppose only local identification as in some industrial systems around specific operating point. Compared to methods that could detect both operating conditions evolution and detect, isolate and estimate faults in nonlinear system as proposed by [12] and [15], this paper considers time varying modeling errors. Thus, the main contribution of this paper consists in the synthesis of a residual generator based on a Polytopic Unknown Input Observer $(\mathcal{P U I O})$ which generates robust residual against error modeling under fault occurrence. Another kind of $\mathcal{U I O}$ was developed in [16] for communication purposes but not for fault diagnosis and more over we would like to consider possible multiple different unknown inputs matrices which can be re-designed in a unique matrix in order to ensure fault detection during models transitions.

The paper is organized as follows:

Section II deals with the nonlinear system representation under a convex set through a multi-model approach with taking into account modeling errors in the state space representation. Section III presents an optimal representation of modeling errors and the synthesis of the $\mathcal{P U I O}$. In Section IV, stability of the $\mathcal{P U I O}$ is performed by use of Linear Matrix Inequalities $(\mathcal{L M I})$ for observer gain synthesis. In the last Section, the performances of the $\mathcal{P U I O}$ are illustrated through a nonlinear CSTR simulation.

\section{NONLINEAR REPRESENTATION AND FDI FOR POLYTOPIC SYSTEMS}

\section{A. Nonlinear Representation}

Consider a discrete-time nonlinear dynamical system in fault-free case described by:

$$
\left\{\begin{array}{c}
x_{k+1}=g\left(x_{k}, u_{k}\right) \\
y_{k}=h\left(x_{k}, u_{k}\right)
\end{array}\right.
$$

where $x_{k} \in \mathcal{X} \in \mathbb{R}^{n}$ represents the state vector, $u_{k} \in$ $\mathcal{U} \in \mathbb{R}^{p}$ is the input vector and $y \in \mathbb{R}^{m}$ is the output vector. We assume that functions $g\left(x_{k}, u_{k}\right)$ and $h\left(x_{k}, u_{k}\right)$ are continuously differentiable. 
It is assumed that dynamic behaviour of the system operating at different operating points can be approximated by a set of $N$ linear time invariant models. Consider the following state space representation of a nonlinear system around i-th operating point, $\forall i \in[1, \ldots, N]$, with additive actuator faults:

$$
\begin{aligned}
& x_{k+1}-x_{e}^{i}=A_{i}\left(x_{k}-x_{e}^{i}\right)+B_{i}\left(u_{k}-u_{e}^{i}\right)+E_{i} d_{k}+F_{i} f_{k} \\
& y_{k}-y_{e}^{i}=C_{i}\left(x_{k}-x_{e}^{i}\right)+D_{i}\left(u_{k}-u_{e}^{i}\right)
\end{aligned}
$$

Matrices $\left(A_{i}, B_{i}, C_{i}, D_{i}\right)$ are invariant matrices defined around the $i^{\text {th }}$ operating point $\left(\mathcal{O P} \mathcal{P}_{i}\right)$ generally obtained from a nonlinear system using a first-order Taylor expansion around $\left(x_{e}^{i}, u_{e}^{i}\right)$ with $y_{e}^{i}=C_{i} x_{e}^{i}+D_{i} u_{e}^{i}$ or identification of a nonlinear system around predefined operating points as for example in chemical processes in [11] and [10]. The fault distribution matrix is represented by $F_{i} \in \mathbb{R}^{n \times p}$. $f_{k} \in \mathbb{R}^{p}$ represents the actuator fault vector and in faultfree case it is obviously equal to zero. The modeling errors matrix are noted as $E_{i} \in \mathbb{R}^{n \times q}$ and are supposed to be full column rank for each operating point and $d_{k} \in \mathbb{R}^{q}$ is the associated modeling error vector. These matrices $E_{i}$ can be obtained from a second-order Taylor expansion or through an identification procedure as treated in [1]. In the following, we consider that $D_{i}=0$. As proposed in fault-free case in [7], [9] and in faulty-case in [17](where $E_{i}$ was not considered), (2) can be specified by the set of system matrices:

$$
M_{i}=\left[\begin{array}{llll}
A_{i} & B_{i} & E_{i} & F_{i} \\
C_{i} & & &
\end{array}\right], \forall i \in[1 \ldots N]
$$

Let $S_{k}$ be a matrix sequence varying within a convex set, defined as:

$$
M_{k}:=\left\{\sum_{i=1}^{N} \rho_{k}^{i} M_{i}: \rho_{k}^{i} \geq 0, \sum_{i=1}^{N} \rho_{k}^{i}=1\right\}
$$

So, $M_{k}$ characterizes at each sample the nonlinear system from an $\mathcal{O P}$ to another. Consequently, the dynamic behavior of nonlinear system can be defined by a convex set of a multiple LTI models, noted $\Upsilon\left(\Upsilon:=\left\{\left[M_{1}, \ldots, M_{N}\right]\right\}\right)$.

The representation (4) under a convex set can be considered as a conventional modeling approach for nonlinear smooth plant where $\rho_{k}^{i}$ is an appropriate weighting function. The function $\rho_{k}^{i}$ embodies the nonlinearity of the plant and $\rho_{k}^{i} \quad \forall i \in[1 \ldots N]$ lie in a convex set $\Omega=\left\{\rho_{k}^{i} \in \mathbb{R}^{N}, \rho_{k}=\right.$ $\left[\begin{array}{ll}\rho_{k}^{1} & \ldots . \rho_{k}^{N}\end{array}\right]^{T}, \quad \rho_{k}^{i} \geq 0 \quad \forall i \quad$ and $\left.\quad \sum_{i=1}^{N} \rho_{k}^{i}=1\right\}$ and these functions are directly generated via works of [15] and [12], which permit to generate insensitive residual to faults. Under the assumptions that these weighting functions $\rho_{k}^{i}$ are considered as scheduling variables which are not affected by faults or modeling errors as proposed by [15] and [12], the nonlinear system (1) can be described by the following state space representation:

$$
\begin{aligned}
x_{k+1} & =\sum_{i=1}^{N} \rho_{k}^{i}\left[A_{i} x_{k}+B_{i} u_{k}+E_{i} d_{k}+F_{i} f_{k}+\Delta x_{i}\right] \\
y_{k} & =\sum_{i=1}^{N} \rho_{k}^{i}\left[C_{i} x_{k}\right]
\end{aligned}
$$

with the term $\Delta x_{i}$, around an $i$ th operating point, equals to:

$$
\Delta x_{i}=-A_{i} x_{e}^{i}-B_{i} u_{e}^{i}+x_{e}^{i}
$$

As referred in (3), a new set of matrices is designed with the additional term $\Delta x_{i}$ such that:

$$
S_{i}=\left[\begin{array}{ccccc}
A_{i} & B_{i} & E_{i} & F_{i} & \Delta x_{i} \\
C_{i} & & & &
\end{array}\right], \forall i \in[1 \ldots N]
$$

Based on the representation (5), we focus our attention on the synthesis of the Polytopic Unknown Input Observer. In order to realize an efficient FDI, a decoupled residual against modeling errors should be generated and modeling errors matrices definition must be reconsidered.

\section{MODELING ERRORS REPRESENTATION}

Modeling errors $E_{i}$ are likely different for each operating point with different distribution matrices. One way to achieve a fault diagnosis robust to modeling errors is to make disturbance de-coupling conditions hold true (in an optimal sense) for all disturbance distribution matrices. This can be done by using a single optimal disturbance distribution matrix $E^{*}$ to approximate all of them as proposed by [1]. This optimal matrix $E^{*}$ with full column rank makes possible de-coupling residual from modeling errors between different $\mathcal{O P}$. An exact de-coupling for each $\mathcal{O P}$ is robust against modeling errors around the $\mathcal{O P}$ but not during transitions between the different $\mathcal{O P}$.

Let consider a set $R$ which contains $N$ modeling errors modeling matrices such that

$$
\left[E_{1} E_{2} \ldots E_{N}\right]=R
$$

An optimal matrix $E^{*}$ that can approximate the set $R$ is equivalent to the following optimization problem:

$$
\begin{aligned}
& \min _{R^{*}}\left\|R-R^{*}\right\|_{F}^{2} \\
& \text { subject to } \operatorname{rank}\left(R^{*}\right) \leq m
\end{aligned}
$$

where $\|.\|_{F}^{2}$ denotes the Frobenius norm. The matrix $R^{*}$ is thus chosen so that the sum of the squared distances between the columns of $R$ and $R^{*}$ is minimized subject to the constraint $R^{*} \leq m$. Moreover, the rank condition is due to the fact that FDI scheme can distinguish faults and modeling errors with all most $m$ independent outputs. This optimization problem can be solved by using Singular Value Decomposition (SVD) on the set $R^{*}$.

The second step is to obtain the required distribution matrix $E^{*}$ with appropriate dimension by decomposing the rank deficient matrix $R^{*}$ as:

$$
R^{*}=R_{1} R_{2}
$$

where $R_{1} \in \mathbb{R}^{n \times q}$ is full column rank and $R_{2} \in \mathbb{R}^{q \times(N \times q)}$. An optimal approximation for the modeling errors distribution matrix is $R_{1}$ with rank $q$ equivalent to matrix $E^{*}$.

Consequently, the following state space representation could be considered to describe the dynamic behaviour of the nonlinear system:

$$
\begin{aligned}
x_{k+1} & \simeq \sum_{i=1}^{N} \rho_{k}^{i}\left[A_{i} x_{k}+B_{i} u_{k}+E^{*} d_{k}+F_{i} f_{k}+\Delta x_{i}\right] \\
y_{k} & =C x_{k}
\end{aligned}
$$

Moreover, it is often possible to consider only one output matrix $C$ if there is no nonlinearity on the outputs. 
So, based on equation (11), a robust polytopic unknown input observer against modeling errors is developed in the next section.

\section{Polytopic Unknown Input ObServer Design}

Definition 1: (Polytopic Unknown Input Observer) A polytopic observer is defined as a polytopic unknown input observer for the system described by (11) without fault $\left(f_{k}=0\right)$, if the estimation error tends asymptotically to zero despite unknown disturbances on the system.

This polytopic unknown input observer is defined such that:

$$
\begin{aligned}
z_{k+1} & =\sum_{i=1}^{N} \rho_{k}^{i}\left[S_{i} z_{k}+T B_{i} u_{k}+K_{i} y_{k}+\Delta z_{i}\right] \\
\widehat{x}_{k+1} & =z_{k+1}+H^{*} y_{k+1}
\end{aligned}
$$

where $\widehat{x}$ is the state space estimation of $x$. The estimation error is equivalent to

$$
\begin{aligned}
e_{k+1} & =x_{k+1}-\widehat{x}_{k+1} \\
& =x_{k+1}-\left(z_{k+1}+H^{*} y_{k+1}\right) \\
& =x_{k+1}-H^{*}\left(C x_{k+1}\right)-z_{k+1} \\
& =\left[I-H^{*} C\right] x_{k+1}-\left(S(\rho) z_{k}+T B(\rho) u_{k}\right. \\
& \left.+K(\rho) y_{k}+\Delta z(\rho)\right)
\end{aligned}
$$

with notation $().(\rho)$ stands for $\sum_{i=1}^{N} \rho_{k}^{i}(\cdot)_{i}$. By taking into account gain decomposition $K(\rho)$ such as $K(\rho)=K^{1}(\rho)+$ $\Pi(\rho)$, the equation (12) leads to:

$$
\begin{aligned}
e_{k+1} & =\left[I-H^{*} C\right]\left(A(\rho) x_{k}+B(\rho) u_{k}+E^{*} d_{k}+F(\rho) f_{k}\right. \\
& +\Delta x(\rho))-\Delta z(\rho)-T B(\rho) u_{k} \\
& -S(\rho)\left(x_{k}-e_{k}-H^{*} y_{k}\right)-K^{1}(\rho) C x_{k}-\Pi(\rho) y_{k}
\end{aligned}
$$

The estimation error is equivalent to:

$$
\begin{aligned}
e_{k+1} & =S(\rho) e_{k}-\left[S(\rho)-\left(I-H^{*} C\right) A(\rho)-K^{1}(\rho) C\right] x_{k} \\
& +\left(I-H^{*} C\right) E^{*} d_{k}-\left[\Pi(\rho)-S(\rho) H^{*}\right] y_{k} \\
& +\left[I-H^{*} C\right] \Delta x(\rho)+\left(I-H^{*} C\right) F(\rho) f_{k} \\
& -\Delta z(\rho)-\left[T-\left(I-H^{*} C\right)\right] B(\rho) u_{k}
\end{aligned}
$$

$S(\rho), T, K(\rho)$ and $H^{*}$ are designed so as to ensure the stability and the convergence of the estimation error $e_{k}=$ $x_{k}-\widehat{x}_{k}$ without fault on the system $\left(f_{k}=0\right)$. To obtain an exact decoupling, based on an optimal modeling errors distribution matrix $E^{*}$, the following conditions should be satisfied:

$$
\begin{aligned}
& S(\rho)=T A(\rho)-K^{1}(\rho) C \quad \Pi(\rho)=S(\rho) H^{*} \\
& \left(I-H^{*} C\right) E^{*}=0 \quad T=I-H^{*} C \quad T \Delta x(\rho)=\Delta z(\rho)
\end{aligned}
$$

The robust polytopic $\mathcal{U I O}$ design is realized when equations (15) hold true and $S(\rho)$ is stable. So, estimation error without fault occurrence, denoted $\bar{e}_{k}$, tends asymptotically to zero if all these conditions (15) are satisfied. The gain design $K^{1}(\rho)$ is addressed in the next section. The necessary and sufficient conditions for the existence of a polytopic $\mathcal{U I O}$ are:

(i) $\operatorname{Rank}\left(C E^{*}\right)=\operatorname{Rank}\left(E^{*}\right)$

(ii) $\left(T A_{i}, C\right)$ are detectable pairs, $\forall i \in[1,2, \ldots, N]$. These conditions are related in linear case [1].
If condition $(i)$ is true, the synthesis of matrix $H^{*}$, which can permit to avoid unknown input effects, is performed by:

$$
H^{*}=E^{*}\left(C E^{*}\right)^{+}
$$

It should be noted that the matrix $E^{*}$ is a constant matrix $\forall i \in[1,2, \ldots, N]$. If conditions (15) hold true, the estimation error $e_{k}$ and the residual $r_{k}$ are described as:

$$
\begin{aligned}
& e_{k+1}=S(\rho) e_{k}+\left(I-H^{*} C\right) F(\rho) f_{k} \\
& r_{k}=C e_{k}
\end{aligned}
$$

Moreover, condition (ii) ensures that a gain $K^{1}(\rho)$ can be synthesized in order to obtain a Hurwitz matrix $S(\rho)=$ $T A(\rho)-K^{1}(\rho) C$ and an estimation error which tends to zero without fault. $E^{*}$ is a fixed matrix over the operating range and for detection and isolation purposes, it is important to constraint $q+f<m$, i.e. the rank $q$ of modeling errors matrix and the rank $f$ of the faulty distribution matrix have to be smaller than the number $m$ of the outputs. For an actuator fault isolation, a bank of $p$ polytopic $\mathcal{U I O}$ has to be used where each polytopic $\mathcal{U I O}$ is computed with a different

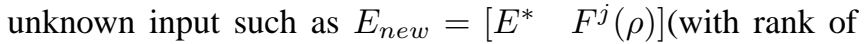
$E_{\text {new }}$ equals to $\left.q+f\right)$, with $F^{j}(\rho)$ is the $j$ th column of matrix $F(\rho)$.

If (15) and (16) are fulfilled, the estimation error $e_{k}$ and residual $r_{k}$ lead to:

$$
\begin{aligned}
& e_{k+1}=S(\rho) e_{k}+T F(\rho) f_{k} \\
& r_{k}=C e_{k}
\end{aligned}
$$

with an exact de-coupling on $E^{*}$. However, it is essential to examine faulty distribution matrices $F_{i}$ with modeling error matrix $E^{*}$ which have to generate different spaces. Indeed, de-coupling can reject perturbations through the term $T E^{*}$ in equation (15), whereas fault are represented by the term $T F(\rho)$. If it is very important in FDI scheme to cancel perturbation on a system, it is widely important to be able to detect and isolate fault through estimation error $e_{k}$ and $r_{k}$. Thus, some relations have to be verified around all the operating points:

$$
\operatorname{Rank}[T F(\rho)]=\operatorname{rank}[F(\rho)], \forall \rho \in \Omega
$$

This condition allows to verify that de-coupling do not affect fault detection by the estimation error. This condition can be translated in a geometric way such as:

$$
\bigcup_{i=1}^{N} \operatorname{Im}\left(F_{i}\right) \subseteq \operatorname{Im}\left(T^{T}\right)
$$

If the union image $F_{i}$ is included in the image of decoupling matrix $T^{T}$, then fault detection is possible over all the operating range. It is equivalent to:

$$
\begin{aligned}
& \bigcup_{i=1}^{N} \operatorname{Im}\left(F_{i}\right) \bigcap \operatorname{Ker}(T)=\{0\} \\
& =\operatorname{Im}\left(\left[F_{1} \ldots F_{i} \ldots F_{N}\right]\right) \bigcap \operatorname{Ker}(T)=\{0\}
\end{aligned}
$$

If condition (21), which can be checked off-line, is fulfilled, de-coupling do not affect fault detection. It could be noticed 
that all $F_{i}$ are supposed to be full column rank in order to avoid fault compensation of non-independent columns. The estimation error is not corrupt by modeling errors based on an approximated matrix $E^{*}$ over the all operating range; but there should remain a little difference due to the minimization criterion (9).

\section{GAIN SYNTHESIS BY $\mathcal{L} \mathcal{M} \mathcal{I}$}

\section{A. Introduction on $\mathcal{L} \mathcal{M I}$ Region}

Pole assignment by $\mathcal{L} \mathcal{M I}$ is dedicated for convex sets [18]. The main objective is to ensure, at first, polytopic observer stability and in a second time to constraint its poles in a specified region of the complex plane. The observer dynamic is performed by decomposing complex plane in $\mathcal{L} \mathcal{M I}$ region.

Definition 2: $\mathcal{L} \mathcal{M I}$ region [19]

A subset $\mathcal{D}$ of the complex plane is called an $\mathcal{L} \mathcal{M I}$ region if there exists a symmetric matrix $\alpha \in \mathbb{R}^{m \times m}$ and a matrix $\beta \in \mathbb{R}^{m \times m}$, such that

$$
\mathcal{D}=z \in \mathcal{C}: f_{\mathcal{D}}(z)<0
$$

with $f_{\mathcal{D}}(z)=\alpha+z \beta+\bar{z} \beta^{T}$ where $f_{\mathcal{D}}(z)$ is called the characteristic function.

Eigenvalues of a real matrix $A$ lie in $\mathcal{D}$ [19], if and only if there exists a symmetric positive definite matrix $P>0$, such that

$$
M_{\mathcal{D}}(A, P)=\alpha \otimes P+\beta \otimes(A P)+\beta^{T} \otimes(A P)^{T}<0
$$

Then, it is both possible to constraint poles of the observer to a prescribed region and ensure it stability.

\section{B. Poles Placement of the Robust Polytopic $\mathcal{U I O}$ under $\mathcal{L} \mathcal{M I}$ Constraints}

According to (18), the estimation error $\bar{e}_{k}$, without fault, is expressed as

$$
\begin{aligned}
\bar{e}_{k+1} & =S(\rho) \bar{e}_{k}=\left(\left(I-H^{*} C\right) A(\rho)-K^{1}(\rho) C\right) \bar{e}_{k} \\
& =\left(\bar{A}(\rho)-K^{1}(\rho) C\right) \bar{e}_{k}
\end{aligned}
$$

Proposition 1: Let $\mathcal{D}(r)$ a $\mathcal{L} \mathcal{M I}$ region defining a disk (included in the unit circle) with a center $(0,0)$ and a radius $0<r<1$. The estimation error (24) is called quadratically $\mathcal{D}$-stabilizable (all the complex poles lie in $\mathcal{L} \mathcal{M I}$ region $\mathcal{D}$ ) if there exists matrices $R_{i}$ for $i=[1 \ldots N]$ and a positive symmetric matrix $X>0$ with appropriate dimensions such that $\forall i \in[1 \ldots N]$ :

$$
\left(\begin{array}{cc}
-r X & \bar{A}_{i}^{T} X-C^{T} R_{i}^{T} \\
X \bar{A}_{i}-R_{i} C & -r X
\end{array}\right)<0
$$

with $R_{i}=X K_{i}^{1}$.

Proof:

If (25) is fulfilled $\forall i \in[1 \ldots N]$, then by using notation $R_{i}=X K_{i}^{1}$, it follows that

$$
\left(\begin{array}{cc}
-r X & \left(\bar{A}_{i}-K_{i}^{1} C\right)^{T} X \\
X\left(\bar{A}_{i}-K_{i}^{1} C\right) & -r X
\end{array}\right)<0
$$

The poles of the matrix $\left(\bar{A}_{i}-K_{i}^{1} C\right)$ lie in a circle with a center $(0,0)$ and a radius $0<r<1$ (Cf. Definitions of such elementary $\mathcal{L} \mathcal{M I}$ regions in [19]). By weighting each $\mathcal{L} \mathcal{M I}$
(26) by $\rho_{i}$ and summing all of them with $\sum_{i=1}^{N} \rho_{i}=1$, the inequality (26) leads to

$\left(\begin{array}{cc}-r X & \sum_{i=1}^{N} \rho_{i}\left(\bar{A}_{i}-K_{i}^{1} C\right)^{T} X \\ X \sum_{i=1}^{N} \rho_{i}\left(\bar{A}_{i}-K_{i}^{1} C\right) & -r X\end{array}\right)_{(27)}<0$

where matrix $\sum_{i=1}^{N} \rho_{i}\left(\bar{A}_{i}-K_{i}^{1} C\right)$ can be written as $(\bar{A}(\rho)-$ $\left.K^{1}(\rho) C\right)$ under the convex set $\Omega$, ensuring that poles of the estimation error $\bar{e}_{k}$ in (24) lie in region $\mathcal{D}$ with gains $K^{1}(\rho)=\sum_{i=1}^{N} \rho_{i} X^{-1} R_{i}, \forall i \in[1, \ldots, N]$. The estimation is said quadratic $\mathcal{D}$-stable.

Remark: if parameter $r=1$ then $\mathcal{L} \mathcal{M I}$ in (25) expresses quadratic stability in discrete case with notation $R_{i}=X K_{i}^{1}$ and with a Schür Complement, we find after some computations:

$\left(\bar{A}_{i}-K_{i}^{1} C\right)^{T} P\left(\bar{A}_{i}-K_{i}^{1} C\right)-P<0$ and $P>0$ that is the quadratic stability of estimation error with respect to $X^{-1}=P>0$ at each vertex $\forall i \in[1, \ldots, N]$.

We should note that using $\mathcal{L} \mathcal{M I}$, observer gain synthesis is made through a polytopic form and estimation error (24) satisfies to the condition of quadratic $\mathcal{D}$-stability with $X>0$ for the Lyapunov matrix. This proposed solution allows to ensure a quadratic $\mathcal{D}$-stability for the estimation error (without fault) and to fixe the dynamic of the polytopic $\mathcal{U I O}$ for the considered system. Poles constraints are specified with $\mathcal{L} \mathcal{M I}$ defining a prescribed region of the complex plan.

\section{CSTR: CONTINUOUS STIRRED TANK REACTION}

\section{A. System Description}

The performance of the robust polytopic $\mathcal{U I O}$ is illustrated through a Continuous Stirred Tank Reaction simulation (CSTR). A full description of the CSTR could be found in [20]. This system is a nonlinear chemical reactor that exhibits many interesting properties like input multiplicity, a gain sign change, asymmetric response and both minimum and nonminimum phase behavior [21]. The, so called van der Vusse reaction, is summarized by the following reaction scheme: $A \Rightarrow B$ with $B \Rightarrow C$ and $2 A \Rightarrow D$.

The dynamic behaviour of the reactor is classically described by the following nonlinear differential equations:

$$
\left\{\begin{aligned}
\dot{C}_{A} & =\frac{\dot{V}}{V_{r}}\left(C_{A 0}-C_{A}\right)-k_{1}(v) C_{A}-k_{3}(v) C_{A}^{2} \\
C_{B} & =\frac{\dot{V}}{V_{r}} C_{B}+k_{1}(v) C_{A}-k_{2}(v) C_{B} \\
\dot{v} & =\frac{\dot{V}}{V_{r}}\left(v_{0}-v\right)-\frac{1}{\rho C_{p}}\left(k 1(v) C_{A} \Delta H_{R A B}+k 2(v) C_{B}\right. \\
& \left.\times \Delta H_{R B C} k 1(v)+k_{3}(v) C_{A}^{2} \Delta H_{R A D}\right)+\frac{k_{w} A_{r}}{\rho C_{p} V_{r}}\left(v-v_{k}\right) \\
\dot{v_{k}} & =\frac{1}{m_{k} C_{P K}} \times\left(\left(\dot{Q}_{k}-v_{k}\right)+k_{w} A_{r}\left(v-v_{k}\right)\right)
\end{aligned}\right.
$$

with $k_{i}(v)=k_{i 0} e^{\frac{E_{i}}{v+273.15}}$. The model has four states: concentration of $\mathrm{A}\left(C_{A}\right)$ with an initial concentration $C_{A 0}$, concentration of $\mathrm{B}\left(C_{B}\right)$, temperature $v$ with a fixed value for the feed temperature $v_{0}$ and cooling jacket temperature $v_{k}$. It is assumed that states are directly measurable except for $v_{k}$. The input variables are the flow rate normalized by the reactor volume $\frac{\dot{V}}{V_{r}}$ and the heat removal $\dot{Q}_{k}$.

\section{B. Operating Conditions}

A specific condition, reported in the benchmark [20], underlines the fact that concentration of $\mathrm{B}$ should be taken 
into the range $[0.8 ; 1.09]$ mol. $^{-1}$ for manipulated variables $\frac{\dot{V}}{V_{r}}$ lying in $\left[3 h^{-1} ; 35 h^{-1}\right]$ and $\left[-9000 k J . h^{-1} ; 0\right]$ for $\dot{Q}_{k}$. The feed temperature $v_{0}$ is considered as a fixed value at $105^{\circ} \mathrm{C}$ as proposed in [20]. In the proposed study, the CSTR is controlled in an open-loop way with multiple desirable concentrations of product $\mathrm{B}$, each of them defining an operating point. Thus, CSTR could produce different concentrations $C_{B}$ and the system is described around predefined operating points. By taking into account the static characteristic illustrated in figure (1) and in order to exploit the nonlinear system in a wide range, concentrations of $\mathrm{B}$ are $C_{B 1}, C_{B 2}, C_{B 3}$ with respectively $0.85,1.00,1.09 \mathrm{~mol} \mathrm{~L}^{-1}$.

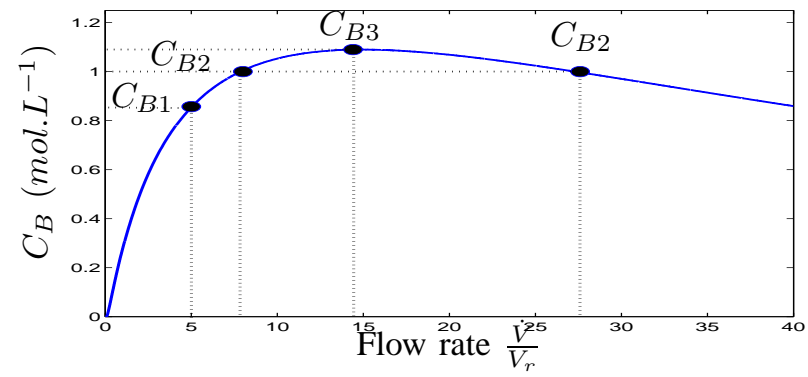

Fig. 1. Steady state gains

It can be noted that concentration $C_{B 2}=1.00 \mathrm{~mol} . \mathrm{L}^{-1}$ could be obtained for two different control values $\frac{\dot{V}}{V_{r}}$ : two operating points are associated to this concentration.
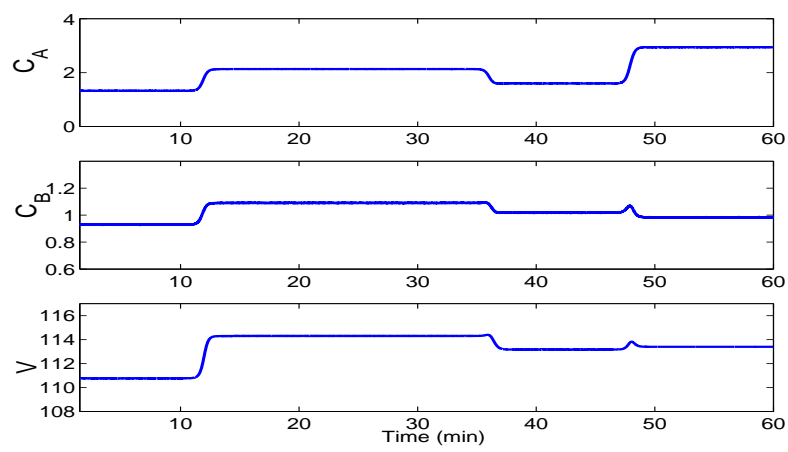

Fig. 2. Dynamic evolution of the measurable states

Figure (2) shows the dynamic evolution of the measurable states with a flow rate, in the nominal case, presented in figure (4) that permits to cover the wide operating range conditions. It could be noticed that there is non-minimum phase behaviour appearing on concentrations of $A$ and $B$ during a transition.

\section{Experiments and Results}

Based on the physical dynamic equation of the system, linear state space models were obtained around four $\mathcal{O P}$ using a first order linearization. These four operating points developed are: $C_{B 1}=0.85 \mathrm{~mol} . \mathrm{L}^{-1}, C_{B 3}=1.09 \mathrm{~mol} . \mathrm{L}^{-1}$ and $C_{B 2}=1.00 \mathrm{~mol} . \mathrm{L}^{-1}$ with $\frac{\dot{V}_{21}}{V_{r}}=7.8570 \mathrm{~h}^{-1}$ and $C_{B 2}=1.00$ mol. $L^{-1}$ with $\frac{\dot{V}_{22}}{V_{r}}=27.90 h^{-1}$. The nonlinear model has been discretized through a Tustin method in order to provide discrete linear models represented in equation (11). Disturbance matrices of modeling error $E_{i}$ are directly obtained by a second order linearization and an unique matrix $E^{*}$ is computed through an optimization technique based on Singular Value Decomposition as proposed in [1]. An unique actuator fault on the manipulated variables $\frac{\dot{V}}{V_{r}}$ is considered, fault matrices $F_{i}$ are related with the column of $B_{i}$. According to the four linear models and under the assumptions that the interpolation functions $\rho_{i}$ are insensitive to faults , figure (3) shows the sequence of activation functions $\rho_{i}$ generated from [17].

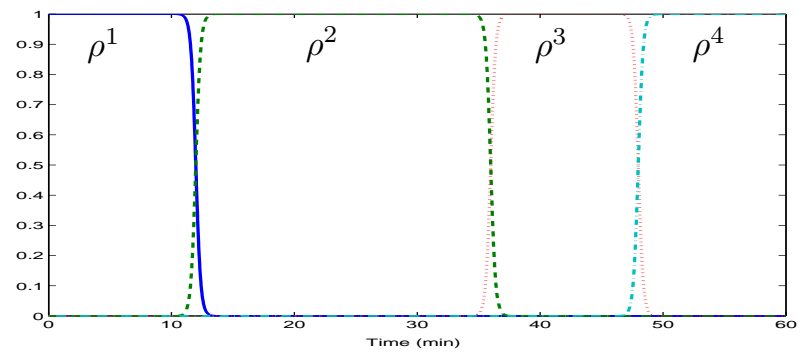

Fig. 3. Weighting functions

Based on the unique disturbance matrice $E^{*} \in \mathbb{R}^{4 \times 2}$, the polytopic observer gain synthesis is performed by pole placement developed in the previous paragraph by a $\mathcal{L} \mathcal{M I}$ region defined with $r=0.5$. The different matrices are given in the Appendix.

In a first experiment, the polytopic $\mathcal{U I O}$ is evaluated without fault with the control input illustrated in figure (4). This residual norm is presented in figure (5). It can be observed that this norm is different from zero around operating points because polytopic $\mathcal{U I O}$ is synthesized in order to obtain an exact decoupling against $E^{*}$, but this matrix is just an optimal approximation of modeling errors. During transitions, uncertainties are minimized and the norm is very closed to zero. The magnitude of the residual norm is almost constant through all operating points and represents the modeling errors due to approximations of modeling error representation. We should underline that an adaptive threshold could be taken into account the specificities of the polytopic $\mathcal{U I O}$ synthesis in order to generate an efficient residual evaluation.

In a second experiment, an actuator fault with a loss of $65 \%$ from the nominal value, is introduced as in figure (4) during the second and the third operating point. The faulty control input is sent to the system and the computed control input is exploited by the polytopic $\mathcal{U I O}$. This experiment simulates an actuator fault that would be stuck between $\mathcal{O P} \mathcal{P}_{1}$ and $\mathcal{O P}$, then, a partial lost of an actuator fault is simulated during transitions of $\mathcal{O P}_{2}$ and $\mathcal{O P}_{3}$. At the last change of operating point $\mathcal{O P}_{3}-\mathcal{O P}_{4}$, the actuator fault disappears. Due to the fact that we consider only one actuator fault which can occur into the system, the fault detection is equivalent to a fault isolation result. According to the synthesis of the robust polytopic $\mathcal{U I O}$, the residual norm is sensitive to the fault occurrence. As illustrated in figure (5), the residual norm of 


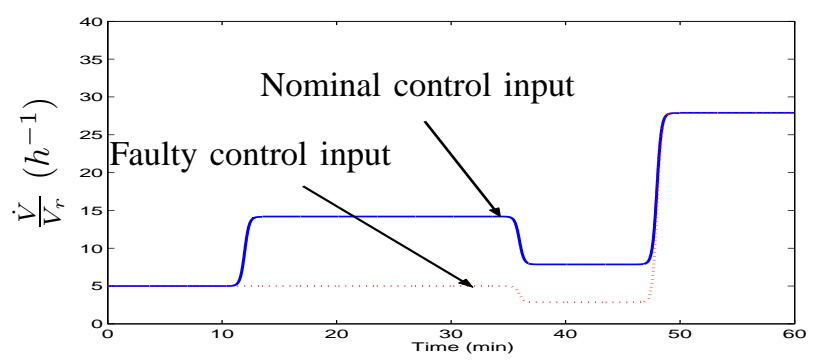

Fig. 4. Faulty control input and nominal control input

the polytopic $\mathcal{U I O}$ is sensitive to actuator fault and robust to modeling errors. The actuator fault is detected easily due to the magnitude of the residual norm.

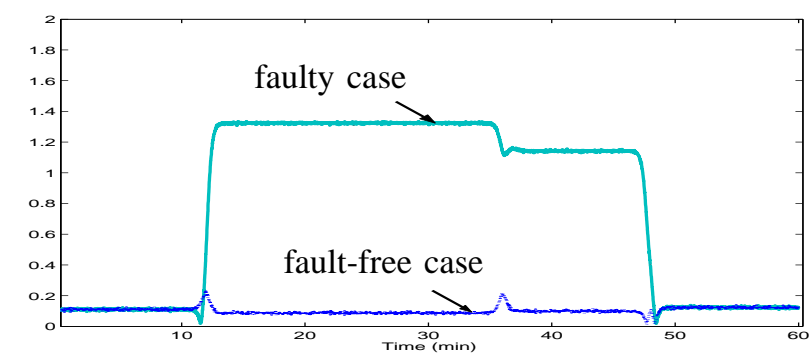

Fig. 5. Residual norms evolution with and without fault

\section{CONCLUSION}

In this paper, a new residual generator robust against modeling errors was developed. This residual generation is dedicated to fault diagnosis of systems based on a multimodel approach which operate around specific and predefined $\mathcal{O P}$. The modelling errors approximation over a set of $\mathcal{O P}$ allows an unique decoupling for all the system range even during transitions. Our contribution is underlined by a polytopic $\mathcal{U I O}$ that generates decoupled residuals from modeling errors. The observer gain synthesis is realized by $\mathcal{L M I}$ so as to ensure both stability and pole assignment of the polytopic $\mathcal{U I O}$. Actuator fault diagnosis of nonlinear systems is realized under modeling errors. The illustration is made through a CSTR simulation around multi operating conditions.

$$
\begin{aligned}
& \text { APPENDIX } \\
& E^{*}=\left[\begin{array}{cc}
-0.8142 & 1.3831 \\
-0.0032 & -1.6090 \\
11.7210 & 0.0956 \\
0 & 0
\end{array}\right] H \stackrel{*}{=}\left[\begin{array}{ccc}
0.42 & -0.49 & -0.03 \\
-0.49 & 0.57 & -0.03 \\
-0.03 & -0.03 & 0.99 \\
0 & 0 & 0
\end{array}\right] \\
& K_{1}^{1}=\left[\begin{array}{ccc}
0.43 & 0.51 & 0.03 \\
0.54 & 0.24 & 0.02 \\
0.04 & 0.03 & -0.19 \\
3.78 & 3.27 & 0.79
\end{array}\right] K_{2}^{1}=\left[\begin{array}{ccc}
0.38 & 0.47 & 0.02 \\
0.50 & 0.20 & 0.02 \\
0.04 & 0.03 & -0.19 \\
3.64 & 3.21 & 0.78
\end{array}\right] \\
& K_{3}^{1}=\left[\begin{array}{ccc}
0.41 & 0.50 & 0.03 \\
0.52 & 0.23 & 0.02 \\
0.04 & 0.03 & -0.19 \\
3.81 & 3.25 & 0.79
\end{array}\right] K_{4}^{1}=\left[\begin{array}{ccc}
0.30 & 0.41 & 0.02 \\
0.43 & 0.15 & 0.02 \\
0.03 & 0.02 & -0.19 \\
3.84 & 3.12 & 0.75
\end{array}\right] \\
& X=10^{3} \times\left[\begin{array}{cccc}
1.50 & 0.24 & 0.01 & -0.06 \\
0.24 & 1.42 & 0.01 & -0.05 \\
0.01 & 0.01 & 1.21 & -0.01 \\
-0.06 & -0.05 & -0.01 & 0.01
\end{array}\right] C=\left[\begin{array}{cccc}
1 & 0 & 0 & 0 \\
0 & 1 & 0 & 0 \\
0 & 0 & 1 & 0
\end{array}\right]
\end{aligned}
$$

Matrices $R_{1}, R_{2}, R_{3}, R_{4}$ are not reported. It could be done by the relation $R_{i}=X K_{i}^{1}$.

\section{REFERENCES}

[1] J. Chen and R. Patton, Robust model-based fault diagnosis for dynamic systems. Kluwer Academic Publishers, 1999.

[2] J. Gertler, Fault detection and diagnosis in engineering systems. Marcel Dekker Inc. New York Basel Hong Kong, 1998.

[3] E. Alcorta-Garcia and P. Frank, "Deterministic nonlinear observer based approaches to fault diagnosis: a survey," Control Engineering Practice, vol. 5, no. 5, pp. 663-670, 1997.

[4] M. Kinnaert, "Robust fault detection based on observers for bilinear systems," Automatica, vol. 35(11), pp. 1829-1842, 1999.

[5] C. D. Persis and A. Isidori, "A geometric approach to nonlinear fault detection," IEEE Trans. on Automatic Control, vol. 46(6), pp. 853 $-865,2001$.

[6] H. Hammouri, P. Kabore, and M. Kinnaert, "A geometric approach to fault detection and isolation for bilinear systems," IEEE Trans. on Automatic Control, vol. 46(9), pp. 1451-1455, 2001.

[7] R. Murray-Smith and T. Johansen, Multiple Model Approaches to Modelling and Control. Taylor and Francis, 1997.

[8] D. Angeli, A. Casavola, and E. Mosca, "Constrained predictive control of nonlinear plants via polytopic linear system embedding," International Journal of Robust and Nonlinear Control, vol. 10, pp. 1091$1103,2000$.

[9] A. Tayebi and M. Zaremba, "Iterative learning control for nonlinear systems described by a blended multiple model representation," International Journal of Control, vol. 75, no. 16/17, pp. 1376-1384, 2002.

[10] Z. Wan and M. Kothare, "Efficient scheduled stabilizing model predictive control for constrained nonlinear systems," International Journal of Robust and Nonlinear Control, vol. 13, pp. 331-346, 2003.

[11] L. Ozkan, M. Kothare, and C. Georgakis, "Control of a solution copolymerization reactor using multi-model predictive control," Chemical Engineering Science, pp. 1207-1221, 2003.

[12] D. Theilliol, M. Rodrigues, M. Adam-Medina, and D. Sauter, "Adaptive filter design for FDI in nonlinear systems based on multiple model approach," In proc. IFAC Symposium Safeprocess, Washington D.C, USA, CD Rom, 2003.

[13] P. Maybeck, "Multiple model adaptive algorithms for detecting and compensating sensor and actuator/surface failures in aircraft flight control systems," International Journal of Robust and Nonlinear Control, vol. 9, pp. 1050-1070, 1999.

[14] H. Blom and Y. Bar Shalom, "The interacting multiple model algorithm for systems with Markovian switching coefficients," IEEE Trans. on Automatic Control, vol. 33(8), pp. 780-783, 1988.

[15] M. Adam-Medina, M. Rodrigues, D. Theilliol, and H. Jamouli, "Fault diagnosis in nonlinear systems through an adaptive filter under a convex set representation," In proc. ECC, Cambridge, U.K, CD Rom, 2003.

[16] G. Millerioux and J. Daafouz, "Unknown input observers for messageembedded chaos synchronization of discrete-time systems," International Journal of Bifurcation, vol. 14, pp. 1-12, 2004.

[17] M. Rodrigues, M. Adam-Medina, D. Theilliol, and D. Sauter, "Fault Diagnosis on Industrial Systems based on a Multiple Model approach," In proc. IFAC Symposium on Automation in Mining, Mineral and Metal processing, Nancy, France, 2004.

[18] M. Oliveira, J. Bernussou, and J. Geromel, "A New Discrete-time Robust Stability Condition," Systems and Control Letters, vol. 37, pp. 261-265, 1999.

[19] M. Chilali and P. Gahinet, " $H_{\infty}$ design with pole placement constraints: an LMI approach," IEEE Trans. on Automatic Control, vol. 41, no. 3, pp. 358-367, 1996.

[20] H. Chen, A. Kremling, and F. Allgower, "Nonlinear predictive control of a benchmark cstr," In Proc. 3rd ECC, Italy, pp. 3247-3252, 1995.

[21] E. Gatzke and F. Doyle, "Multiple model approach for CSTR control," World IFAC Congress, Beijing, China, CD Rom, 1999. 